\title{
Repulsion of Single-well Fundamental Edge Magnetoplasmons In Double Quantum Wells
}

\author{
O. G. Balev* and P. Vasilopoulos ${ }^{\dagger}$ \\ ${ }^{*}$ Institute of Semiconductor Physics, National Academy of Sciences, \\ 45 Pr. Nauky, Kiev 252650, Ukraine \\ ${ }^{\dagger}$ Concordia University, Department of Physics, \\ 1455 de Maisonneuve Blvd O, Montréal, Québec, Canada, H3G 1 M8
}

(July 31, 1998)

\begin{abstract}
A microscopic treatment of fundamental edge magnetoplasmons (EMPs) along the edge of a double quantum well (DQW) is presented for strong magnetic fields, low temperatures, and total filling factor $\nu=2$. It is valid for lateral confining potentials that Landau level (LL) flattening can be neglected. The cyclotron and Zeeman energies are assumed larger than the DQW energy splitting $\sqrt{\Delta^{2}+4 T^{2}}$, where $\Delta$ is the splitting of the isolated wells and $T$ the tunneling matrix element. Using a random-phase approximation (RPA), which includes local and nonlocal contributions to the current density, it is shown that for negligible tunnel coupling $2 T \ll \Delta$ the inter-well Coulomb coupling leads to two DQW fundamental EMPs which are strongly renormalized in comparison with the decoupled, single-well fundamental EMP. These DQW modes can be modified further upon varying the inter-well distance $d$, along the $\mathrm{z}$ axis, and/or the separation of the wells' edges $\Delta y$ along the $y$ axis. The charge profile of the fast and slow DQW mode varies, respectively, in an acoustic and optical manner along the $y$ axis and is not smooth on the $\ell_{0}$
\end{abstract}


scale. For strong tunneling $\Delta \lesssim 2 T$ these DQW modes are essentially modified when $\Delta$ is changed by applying a transverse electric field to the DQW.

PACS 73.20.Dx, 73.40.Hm

\section{INTRODUCTION.}

Recently the interest in EMPs has been increased considerably [1]- [17]; for a discussion of older studies see, e.g., Ref. [1]. Initially EMPs were theoretically considered only within essentially classical models [1], [10] in which the edge does not vary but the charge density profile at the edge does. Except for the complications due to the long-range Coulomb interaction, this edge-wave mechanism is the magnetic analog of that for the Kelvin wave |18], i.e., the chiral wave that occurs in tides at the edge of a rotating "shallow" sea, the angular velocity of the sea being the analogue of the cyclotron frequency. More recently a distinctly different, fully quantum mechanical edge-wave mechanism for EMPs was provided by the models of Refs. [4]- [6] and [11], [12]. In these models typically only the edge position

of an incompressible two-dimensional electron gas (2DEG) varies; with respect to that the density profile is that of the undisturbed 2DEG [16].

In Refs. [16]- [17] we proposed a quasi-microscopic model that effectively incorporates both edge-wave mechanisms mentioned above. In this model we treated EMPs $\propto A\left(\omega, q_{x}, y\right) \exp \left[-i\left(\omega t-q_{x} x\right)\right]$, for a single quantum well with $\nu=1$ and very low temperatures $T$, for which the unperturbed density profile drops sharply at the edges on a length of the order of magnetic length $\ell_{0}$. Such a profile is valid for $k_{B} T \ll \hbar v_{g} / \ell_{0}$, where $v_{g}$ is the group velocity of the edge states, and the unperturbed electron density $n_{0}(y)$, normalized to the bulk value $n_{0}$, is calculated as $n_{0}(y) / n_{0}=\left\{1+\Phi\left[\left(y_{r e}-y\right) / \ell_{0}\right]\right\} / 2$, where $y_{r e}$ is the coordinate of the right edge and $\Phi(y)$ the probability integral. However, the current density $j_{\mu}\left(\omega, q_{x}, y\right)$ was obtained [17] when the components of the electric field of the wave, $\mathbf{E}$, are smooth on the $\ell_{0}$ scale. This is not well justified. In addition, nonlocal effects and the screening of the 2DEG were neglected. Later on, within a RPA framework we showed [19] 
that the effect of nonlocality and screening by the 2DEG on the fundamental EMP is small and justifies well, for weak dissipation, the results of Ref. [17 for the fundamental EMP. Here, using the same RPA framework we consider the fundamental EMPs at the edge of a DQW for $\nu=2$, when two occupied Landau sublevels (LSLs), with $n=0$ and the same spin orientation, correspond to different DQW quantum numbers $\alpha_{z}=-$ and $\alpha_{z}=+$. As all studies we are aware of treat EMPs in single quantum well systems, the present microscopic generalization of the model of Refs. [16] and [17] to EMPs in DQWs appears amply justified.

In Sec. II we present the one-electron characteristics of a DQW channel, the wave charge density, inhomogeneous along $y$ on a scale $\sim \ell_{0} \ll \lambda$, and the corresponding electric potential. Further, we derive the integral equations for the fundamental EMPs in a DQW for negligible and strong tunnel coupling. In Sec. III we obtain the corresponding dispersion relations of the fundamental EMPs for very low temperatures. Finally, in Sec. IV we discuss our theory and make concluding remarks.

\section{BASIC RELATIONS}

\section{A. DQW channel characteristics}

We consider a DQW, of width $W$ and of length $L_{x}=L$, in the presence of a strong magnetic field $B$ along the $z$ axis. In the absence of a self-consistent wave potential $V(\mathbf{r}, t)=$ $V\left(\omega_{0}, q_{x}, y, z\right) \exp \left[-i\left(\omega_{0} t-q_{x} x\right)\right]+c . c .$, we assume that the motion of an electron in the 2D-

plane $(x, y)$ is decoupled from it's motion along the $z$ axis. The latter is described by the Schrodinger equation

$$
\hat{h}_{z} X_{\alpha_{z}}(z)=\left[-\frac{\hbar^{2}}{2 m^{*}} \frac{d^{2}}{d z^{2}}+V_{z}\right] X_{\alpha_{z}}(z)=E_{\alpha_{z}} X_{\alpha_{z}}(z)
$$

The DQW consists of two square wells along the $z$ axis, with finite depth $U$, widths $d_{l}$ and $d_{r}$, and separated by a barrier of width $d$. In addition, we assume that $m^{*}$ is independent of $z$. As is usual in DQW studies, we assume that only a pair of tunnel-connected closely located levels, formed from the ground states of the left $(l)$ and right $(r)$ isolated QWs, is essential. 
For small barrier penetration [20] we can use these isolated $r$ and $l$ well eigenfunctions, denoted, respectively, by $X_{r}(z)$ and $X_{l}(z)$ as a basis for solving Eq. (11). With $\alpha_{z}= \pm$ the corresponding normalized orbitals $X_{\alpha_{z}}$ read [20]

$$
X_{+}=N\left[X_{l}+\left(2 T /\left(\Delta+\Delta_{T}\right)\right) X_{r}\right], \quad X_{-}=N\left[X_{r}-\left(2 T /\left(\Delta+\Delta_{T}\right)\right) X_{l}\right] .
$$

Here $N=\sqrt{\left(\Delta+\Delta_{T}\right) / 2 \Delta_{T}}$ is the normalization factor, $T=-T_{0} \exp (-\kappa d)\left(T_{0} \approx\right.$ $2 \pi^{2} \hbar^{2} / m^{*} \kappa d_{l}^{3 / 2} d_{r}^{3 / 2}$ ) is the tunneling matrix element, and $\kappa^{-1} \approx \hbar / \sqrt{2 m^{*} U}$. Further, $\Delta$ is the energy splitting of the isolated $l$ and $r$ levels, and $\Delta_{T}=\sqrt{\Delta^{2}+4 T^{2}}$ is the splitting between the $\alpha_{z}=+$ and $\alpha_{z}=-$ levels; $\Delta_{T}$ takes into account tunnel branch-off. The eigenvalues $E_{+}$and $E_{-}$are $+\Delta_{T} / 2$ and $-\Delta_{T} / 2$, respectively. Here weak barrier penetration means $\exp (-2 \kappa d) \ll 1$ and $\kappa d_{l, r} \gg \pi$. In the calculations of pertinent matrix elements we can approximate $X_{l, r}(z)$ by $\sqrt{2 / d_{l, r}} \cos \left(\pi\left(z-z_{l, r}\right) / d_{l, r}\right)$ for $\left|z-z_{l, r}\right| \leq d_{l, r} / 2$, and by 0 for $\left|z-z_{l, r}\right| \geq d_{l, r} / 2$, where $z_{l}$, and $z_{r}$ are the centers of $l$ and $r$ QWs, respectively. That is, the matrix elements can be calculated without taking into account the barrier penetration of the isolated well orbitals, cf. Ref. [20].

The lateral confining potential is taken flat in the interior of the 2DEG $\left(V_{y}=0\right)$ and parabolic at its edges, e.g., $V_{y}=m^{*} \Omega^{2}\left(y-y_{r}\right)^{2} / 2, y \geq y_{r} . V_{y}$ is assumed smooth on the scale of $\ell_{0}=\left(\hbar / m^{*} \omega_{c}\right)^{1 / 2}$ such that $\Omega \ll \omega_{c}$, where $\omega_{c}=|e| B / m^{*}$ is the cyclotron frequency. We will also consider the case of a tunnel-decoupled DQW, with different confining potentials in the $l$ and $r$ QWs, i.e., $V_{y}^{(l, r)}=m^{*} \Omega^{2}\left(y-y_{r}^{(l, r)}\right)^{2} / 2, y \geq y_{r}^{(l, r)}$ with $y_{r}^{(l)} \neq y_{r}^{(r)}$. In the Landau gauge for the vector potential $\mathbf{A}=(-B y, 0,0)$ and without the interaction $V(\mathbf{r}, t)$, the total one-electron Hamiltonian $\hat{h}^{0}$ is given as $\hat{h}^{0}=\hat{h}_{2 d}+\hat{h}_{z}$, and

$$
\hat{h}_{2 d}=\left[\left(\hat{p}_{x}+e B y\right)^{2}+\hat{p}_{y}^{2}\right] / 2 m^{*}+V_{y}+g^{*} \mu_{B} \hat{S}_{z} B / 2
$$

Here $\hat{\mathbf{p}}$ is the momentum operator, $g^{*}(<0)$ the effective Landé g-factor, and $\mu_{B}$ the Bohr magneton. $\hat{S}_{z}$ is the z-component of the spin operator with eigenvalues $\sigma=1$ and $\sigma=-1$ for spin up $(\uparrow)$ and down $(\downarrow)$, respectively. The eigenvalues and eigenfunctions corresponding to Eq. (3) for the right edge of the channel, where $y_{0} \equiv y_{0}\left(k_{x}\right)=\ell_{0}^{2} k_{x} \geq y_{r}$, are well approximated 21] by 


$$
E_{\alpha_{2 d}} \equiv E_{n, \sigma}\left(k_{x}\right)=(n+1 / 2) \hbar \omega_{c}+m^{*} \Omega^{2}\left(y_{0}-y_{r}\right)^{2} / 2+\sigma g^{*} \mu_{B} B / 2
$$

and

$$
\left|\alpha_{2 d}>\equiv \psi_{\alpha_{2 d}}(\vec{\rho})\right| \sigma>=e^{i k_{x} x} \Psi_{n}\left(y-y_{0}\right) \mid \sigma>/ \sqrt{L}
$$

respectively. Here $\vec{\rho}=\{x, y\}, k_{r}=y_{r} / \ell_{0}^{2}, \alpha_{2 d} \equiv\left\{n, k_{x}, \sigma\right\}, \Psi_{n}(y)$ is a harmonic oscillator function, $\mid \sigma>=\psi_{\sigma}\left(\sigma_{1}\right)=\delta_{\sigma \sigma_{1}}$ is the spin-wave function, and $\sigma_{1}= \pm 1$. Then the eigenvalues of $\hat{h}^{0}$ are $E_{\alpha}=E_{\alpha_{2 d}}+E_{\alpha_{z}}$ and its eigenfunctions $\left|\alpha>=\psi_{\alpha_{2 d}}(\vec{\rho}) X_{\alpha_{z}}(z)\right| \sigma>$.

We will consider only the case of $\nu=2$, when only two tunnel-split levels are occupied. These Landau sublevels (LSLs) have the same Landau and spin quantum numbers, $n=0$ and $\sigma=1$, but different quantum numbers $\alpha_{z}= \pm$. In line with Refs. [22] and [23], we assume that $\hbar \omega_{c}>\Delta_{T}$ and $\left|g^{*}\right| \mu_{B} B>\Delta_{T}$. Then the one-electron energy spectrum (for $\left.y_{0} \geq y_{r}\right)$ for the two occupied LSLs is given by

$$
E_{0, k_{x}, 1, \pm} \equiv E_{0, \pm}\left(k_{x}\right)=\left[\hbar \omega_{c}+m^{*} \Omega^{2}\left(y_{0}-y_{r}\right)^{2}-\left|g^{*}\right| \mu_{B} B \pm \Delta_{T}\right] / 2
$$

Equation (6) leads to the group velocity of the edge states $v_{g 0}^{ \pm}=\hbar^{-1} \partial E_{0, \pm}\left(k_{r}+k_{e}^{0, \pm}\right) / \partial k_{x}=$ $\hbar \Omega^{2} k_{e}^{0, \pm} / m^{*} \omega_{c}^{2}$ with characteristic wave vector $k_{e}^{0, \pm}=\left(\omega_{c} / \hbar \Omega\right) \sqrt{2 m^{*} \Delta_{F 0}^{ \pm}}$, where $\Delta_{F 0}^{ \pm}=$ $E_{F}-\left[\hbar \omega_{c}-\left|g^{*}\right| \mu_{B} B \pm \Delta_{T}\right] / 2>0$, and $E_{F}$ is the Fermi energy. The edge of the LSL is denoted by $y_{r 0}^{ \pm}=y_{r}+\ell_{0}^{2} k_{e}^{0, \pm}=\ell_{0}^{2} k_{r 0}^{ \pm}$, where $k_{r 0}^{ \pm}=k_{r}+k_{e}^{0, \pm}$, and $W=2 y_{r 0}^{-}$. We can also

write $v_{g 0}^{ \pm}=E_{e 0}^{ \pm} / B$, where $E_{e 0}^{ \pm}=\Omega \sqrt{2 m^{*} \Delta_{F 0}^{ \pm}} /|e|$ is the electric field associated with the slope of the confining potential $V_{y}$ at $y_{r 0}^{ \pm}$.

For definiteness, we take the background dielectric constant $\epsilon$ to be spatially homogeneous. Assuming $\left|q_{x}\right| W \gg 1$, we can consider an EMP along the right edge of the channel, of the form $A\left(\omega, q_{x}, y, z\right) \exp \left[-i\left(\omega t-q_{x} x\right)\right]$, totally independent of the left edge.

\section{B. Wave charge density and electric potential at the DQW edge}

As in Refs. [17], [21, we assume that without interaction the one-electron density matrix $\hat{\rho}^{(0)}$ is diagonal, i.e., $\left\langle\alpha\left|\hat{\rho}^{(0)}\right| \beta>=f_{\alpha} \delta_{\alpha \beta}\right.$, where $f_{\alpha}=1 /\left[1+\exp \left(\left(E_{\alpha}-E_{F}\right) / k_{B} T\right)\right]$ is the 
Fermi-Dirac function. In applying the RPA we follow the self-consistent field approach which is discussed, e.g., in Ref. [24]. The one-electron Hamiltonian is $\hat{H}(t)=\hat{h}^{0}+V(x, y, z, t)$ in the presence of the interaction taken as a self-consistent wave potential $V(\mathbf{r}, t)$. Then the equation of motion for the density matrix $\hat{\rho}$ reads

$$
i \hbar \frac{\partial \hat{\rho}}{\partial t}=[\hat{H}(t), \hat{\rho}]-\frac{i \hbar}{\tau}\left(\hat{\rho}-\hat{\rho}^{(0)}\right),
$$

where [,] denotes the commutator. On the right hand side (RHS) of Eq. (17) we have introduced phenomenologically the infinitesimal term $\propto 1 / \tau(\tau \rightarrow \infty)$ that leads to correct rules for passing an integration contour around the pole singularities, etc., cf. Refs. [24][25]. Notice that $\tau \rightarrow \infty$ corresponds to the collisionless case whereas a finite $\tau$ provides the possibility of estimating very roughly the influence of collisions. The most effective scattering is related to intra-LSL and intra-edge transitions [17], [21].

Applying the Laplace transformation over time to Eq. (7) and writing $\hat{R}(\omega)=\int_{0}^{\infty} e^{i \omega t} \hat{\rho} d t$ and $R_{\alpha \beta}(\omega)=<\alpha|\hat{R}(\omega)| \beta>$ we can present the solution of Eq. (7) as a power series in $V$

$$
R_{\alpha \beta}(\omega)=\sum_{m=0}^{\infty} R_{\alpha \beta}^{(m)}(\omega)
$$

where $R_{\alpha \beta}^{(0)}(\omega)=\left(i f_{\alpha} / \omega\right) \delta_{\alpha \beta}$. Because we consider linear EMP's, it is sufficient to take into account only the first two terms $m=0$ and $m=1$ on the RHS of Eq. (8). Then in $V(x, y, z, t)$ we can consider only the term $V\left(\omega_{0}, q_{x}, y, z\right) \exp \left[-i\left(\omega_{0} t-q_{x} x\right)\right]$, which leads to

$$
R_{\alpha \beta}^{(1)}(\omega)=\frac{i\left(f_{\beta}-f_{\alpha}\right)<\alpha\left|V\left(\omega_{0}, q_{x}, y, z\right) e^{i q_{x} x}\right| \beta>}{\left(\omega-\omega_{0}\right)\left[E_{\beta}-E_{\alpha}+\hbar \omega+i \hbar / \tau\right]} .
$$

Because $V(\mathbf{r}, t)$ conserves spin the spin index in Eq. (9) is the same, $\sigma_{\alpha}=\sigma_{\beta}=1$, and therefore it can be dropped. For simplicity we will use the notation $\alpha=\left\{n, k_{x \alpha}, \alpha_{z}\right\}$. Then taking the trace of the density matrix $\hat{\rho}$ with the electron charge density operator, $e \delta(\mathbf{r}-\hat{\mathbf{r}})$, we obtain the wave charge density as

$$
\delta \rho(t, x, y, z) \equiv \rho(t, x, y, z)=\frac{e}{2 \pi} \int_{-\infty+i \eta}^{\infty+i \eta} d \omega e^{-i \omega t} \sum_{\alpha \beta} R_{\alpha \beta}^{(1)}(\omega) \psi_{\beta}^{*}(\mathbf{r}) \psi_{\alpha}(\mathbf{r}),
$$

where $\eta>0$ and $\psi_{\alpha}(\mathbf{r})=\psi_{\alpha_{2 d}}(\vec{\rho}) X_{\alpha_{z}}(z)$. From Eqs. (9) and (10) it follows that $\rho(t, x, y, z)=$ $\rho\left(t, q_{x}, y, z\right) \exp \left(i q_{x} x\right)$. Moreover, for $t / \tau \gg 1$, when contributions related to transitional processes are negligible, Eqs. (9) and (10) lead to $\rho\left(t, q_{x}, y, z\right)=\rho\left(\omega_{0}, q_{x}, y, z\right) \exp \left(-i \omega_{0} t\right)$. 
To simplify the calculations we assume that $d_{l} \rightarrow 0$ and $d_{r} \rightarrow 0$, i.e., we neglect the thickness of both QWs, cf. Ref. 22]. This leads to $X_{l, r}^{2}(z) \rightarrow \delta(z \pm d / 2)$ for $d_{l, r} \rightarrow 0$. Then Poisson's equation gives the wave electric potential $\phi\left(t, q_{x}, y, z\right)$ induced by the density $\rho\left(t, q_{x}, y, z\right)=\rho^{(l)}\left(t, q_{x}, y\right) \delta(z+d / 2)+\rho^{(r)}\left(t, q_{x}, y\right) \delta(z-d / 2)$ in the $l$ QW $(z=-d / 2)$ and $r$ $\mathrm{QW}(z=d / 2)$ as

$$
\phi\left(t, q_{x}, y, z=-d / 2\right)=\phi_{l}\left(t, q_{x}, y\right)=\frac{2}{\epsilon} \int_{-\infty}^{\infty} d y^{\prime} K_{l r}\left(y, y^{\prime} ; t, q_{x}\right)
$$

and

$$
\phi\left(t, q_{x}, y, z=d / 2\right)=\phi_{r}\left(t, q_{x}, y\right)=\frac{2}{\epsilon} \int_{-\infty}^{\infty} d y^{\prime} K_{r l}\left(y, y^{\prime} ; t, q_{x}\right)
$$

where

$$
K_{l r}\left(y, y^{\prime} ; t, q_{x}\right)=K_{0}\left(\left|q_{x}\right|\left|y-y^{\prime}\right|\right) \rho^{(l)}\left(t, q_{x}, y^{\prime}\right)+K_{0}\left(\left|q_{x}\right| d_{y y^{\prime}}\right) \rho^{(r)}\left(t, q_{x}, y^{\prime}\right)
$$

Here $K_{0}(x)$ is the modified Bessel function and $d_{y y^{\prime}}=\sqrt{\left(y-y^{\prime}\right)^{2}+d^{2}}$.

\section{Integral equations for EMP's}

For $t / \tau \gg 1$, we can change $\rho^{(j)}\left(t, q_{x}, y^{\prime}\right)$ to $\rho^{(j)}\left(\omega_{0}, q_{x}, y^{\prime}\right)(j=l, r)$ on the RHS of Eqs. (11) and (12). Then, omitting the common factor $\exp \left(-i \omega_{0} t\right)$, we should change $\phi_{l, r}\left(t, q_{x}, y\right)$ to $\phi_{l, r}\left(\omega_{0}, q_{x}, y\right)$. In the absence of an external potential we have $V\left(\omega_{0}, q_{x}, y, z\right)=$ $e \phi\left(\omega_{0}, q_{x}, y, z\right)$. Then using Eqs. (10)-(12) and integrating Eq. (10) over $z$ once in the neighborhood of $z=-d / 2$ and once in that of $z=d / 2$, we obtain a system of two integral equations for $\rho^{(l)}\left(\omega_{0}, q_{x}, y\right)$ and $\rho^{(r)}\left(\omega_{0}, q_{x}, y\right)$; if we omit the subscript 0 in $\omega_{0}$ they read

$$
\begin{aligned}
\rho^{(j)}\left(\omega, q_{x}, y\right) & =\frac{2 e^{2}}{\epsilon L} \sum_{n_{\alpha}, n_{\beta}=0}^{\infty} \sum_{k_{x \alpha}} \sum_{\alpha_{z}, \beta_{z}} \frac{\left(f_{\beta}-f_{\alpha}\right)<\alpha_{z} \mid \beta_{z}>_{j}}{E_{\beta}-E_{\alpha}+\hbar \omega+i \hbar / \tau} \Pi_{n_{\alpha} n_{\beta}}\left(y, k_{x \alpha}, k_{x \beta}\right) \\
& \times \int_{-\infty}^{\infty} d \tilde{y} \int_{-\infty}^{\infty} d y^{\prime} \Pi_{n_{\alpha} n_{\beta}}\left(\tilde{y}, k_{x \alpha}, k_{x \beta}\right)\left\{\left[<\alpha_{z} \mid \beta_{z}>_{l} K_{l r}\left(\tilde{y}, y^{\prime} ; \omega, q_{x}\right)\right]+[r \leftrightarrow l]\right\}
\end{aligned}
$$

where $\Pi_{n_{\alpha} n_{\beta}}\left(y, k_{x \alpha}, k_{x \beta}\right)=\Psi_{n_{\alpha}}\left(y-y_{0}\left(k_{x \alpha}\right)\right) \Psi_{n_{\beta}}\left(y-y_{0}\left(k_{x \beta}\right)\right), k_{x \beta}=k_{x \alpha}-q_{x},<\alpha_{z} \mid \beta_{z}>_{l}=$ $\int_{-d / 2-0}^{-d / 2+0} d z X_{\alpha_{z}} X_{\beta_{z}}$ and $<\alpha_{z} \mid \beta_{z}>_{r}=\int_{d / 2-0}^{d / 2+0} d z X_{\alpha_{z}} X_{\beta_{z}}$. 
The integral equations (14) take into account the nonlocality in the electron current density $\propto \int d y^{\prime} \sigma_{\mu \nu}\left(y, y^{\prime}\right) E_{\nu}\left(y^{\prime}\right)$, and the screening by the edge and bulk states of the 2DEG. In Ref. 26] it is shown that screening by the edge states can be strong. We consider very low temperatures $T$ satisfying $\hbar v_{g 0}^{ \pm} \gg \ell_{0} k_{B} T$ and use the long-wave length limit $q_{x} \ell_{0} \ll 1$. Further we will obtain only the low-frequency solutions of Eqs. (14) for i) negligibly small tunneling $\omega \ll \omega_{c}$ and ii) strong tunneling $\omega \ll \Delta_{T} / \hbar$. In both cases we assume $\omega_{c}>\Delta_{T} / \hbar$.

Case ii). Comparing the terms $\propto f_{\beta^{*}}$ on the RHS of Eq. (14), for given $n_{\beta^{*}}=0$ and $\beta_{z}^{*}=+$ or - , we find that the contribution to the sum over $n_{\alpha}$ and $\alpha_{z}$ with $n_{\alpha}=n_{\beta^{*}}=0$ and $\alpha_{z}=\beta_{z}^{*}$, is much larger than that of any other term in this sum (e.g., the term with $n_{\alpha}=0$ and $\alpha_{z} \neq \beta_{z}^{*}$ ) or that of all other terms with $n_{\alpha}>0$. The small parameter is $\hbar\left|\omega-q_{x} v_{g 0}^{ \pm}\left(k_{x \beta}\right)\right| / \Delta_{T} \ll 1$, where $v_{g 0}^{ \pm}\left(k_{x \beta}\right)=\hbar^{-1} \partial E_{0, \pm}\left(k_{x \beta}\right) / \partial k_{x \beta}$ is the group velocity of any occupied state $\left\{0, k_{x \beta}, \beta_{z}^{*}\right\}$ of the $\beta_{z}^{*}$ LSL. We assume that both occupied LSLs have one intersection with the Fermi level at the edge of the channel and denote the group velocity of its edge states by $v_{g 0}^{ \pm} \equiv v_{g 0}^{ \pm}\left(k_{r 0}^{ \pm}\right)$. The small parameter given above implies $\hbar q_{x} v_{g 0}^{-} / \Delta_{T} \ll 1$, since $v_{g 0}^{-}$is typically larger than $v_{g 0}^{+}$. Similar results hold for the terms $\propto f_{\alpha^{*}}$ in the sum over $n_{\beta}$ and $\beta_{z}$. Hence for $\hbar \omega \ll \Delta_{T}$ and $\hbar q_{x} v_{g 0}^{ \pm} \ll \Delta_{T}$ the terms with $n_{\alpha} \neq n_{\beta}$ or/and $\alpha_{z} \neq \beta_{z}$ can be neglected. This leads to a system of simpler integral equations for $\rho^{(l)}\left(\omega, q_{x}, y\right)$ and $\rho^{(r)}\left(\omega, q_{x}, y\right)$

$$
\begin{gathered}
\rho^{(j)}\left(\omega, q_{x}, y\right)=\frac{2 e^{2}}{\epsilon L} \sum_{\alpha_{z}, k_{x \alpha}} \frac{<\alpha_{z} \mid \alpha_{z}>_{j}\left(f_{0, k_{x \alpha}-q_{x}, \alpha_{z}}-f_{0, k_{x \alpha}, \alpha_{z}}\right)}{E_{0, \alpha_{z}}\left(k_{x \alpha}-q_{x}\right)-E_{0, \alpha_{z}}\left(k_{x \alpha}\right)+\hbar \omega+i \hbar / \tau} \Pi_{00}\left(y, k_{x \alpha}, k_{x \alpha}-q_{x}\right) \\
\quad \times \int_{-\infty}^{\infty} d \tilde{y} \int_{-\infty}^{\infty} d y^{\prime} \Pi_{00}\left(\tilde{y}, k_{x \alpha}, k_{x \alpha}-q_{x}\right)\left\{\left[<\alpha_{z} \mid \alpha_{z}>_{l} K_{l r}\left(\tilde{y}, y^{\prime} ; \omega, q_{x}\right)\right]+[r \leftrightarrow l]\right\} .
\end{gathered}
$$

Case i). For negligibly small tunneling we have $2|T| / \Delta \ll 1$ and from Eq. (2) it follows that $X_{+}(z) \approx X_{l}(z)$ and $X_{-}(z) \approx X_{r}(z)$. Again we can take $n_{\alpha}=n_{\beta}=0$. Then Eq. (14) for $\omega \ll \omega_{c}, q_{x} v_{g 0}^{ \pm} \ll \omega_{c}, j=l$, and $\alpha_{z}=\beta_{z}=+$ gives

$$
\rho^{(l)}\left(\omega, q_{x}, y\right)=\frac{e^{2}}{\pi \epsilon} \int_{-\infty}^{\infty} d k_{x \alpha} \frac{f_{0, k_{x \alpha}-q_{x},+}-f_{0, k_{x \alpha},+}}{E_{0,+}\left(k_{x \alpha}-q_{x}\right)-E_{0,+}\left(k_{x \alpha}\right)+\hbar \omega+i \hbar / \tau} \Pi_{00}\left(y, k_{x \alpha}, k_{x \alpha}-q_{x}\right)
$$




$$
\times \int_{-\infty}^{\infty} d \tilde{y} \int_{-\infty}^{\infty} d y^{\prime} \Pi_{00}\left(\tilde{y}, k_{x \alpha}, k_{x \alpha}-q_{x}\right) K_{l r}\left(\tilde{y}, y^{\prime} ; \omega, q_{x}\right)
$$

for $j=r$ and $\alpha_{z}=\beta_{z}=-$ the result is

$$
\begin{aligned}
\rho^{(r)}\left(\omega, q_{x}, y\right)= & \frac{e^{2}}{\pi \epsilon} \int_{-\infty}^{\infty} d k_{x \alpha} \frac{f_{0, k_{x \alpha}-q_{x},-}-f_{0, k_{x \alpha},-}}{E_{0,-}\left(k_{x \alpha}-q_{x}\right)-E_{0,-}\left(k_{x \alpha}\right)+\hbar \omega+i \hbar / \tau} \Pi_{00}\left(y, k_{x \alpha}, k_{x \alpha}-q_{x}\right) \\
& \times \int_{-\infty}^{\infty} d \tilde{y} \int_{-\infty}^{\infty} d y^{\prime} \Pi_{00}\left(\tilde{y}, k_{x \alpha}, k_{x \alpha}-q_{x}\right) K_{r l}\left(\tilde{y}, y^{\prime} ; \omega, q_{x}\right) .
\end{aligned}
$$

We will study only the fundamental EMPs following from Eqs. (15)-(17).

\section{FUNDAMENTAL EMPS, $\nu=2$}

\section{A. Negligibly small tunnel coupling}

We now consider case (i), when $2|T| / \Delta \ll 1$ holds, and we have the system of Eqs. (16) and (17) for $\rho^{(l)}\left(\omega, q_{x}, y\right)$ and $\rho^{(r)}\left(\omega, q_{x}, y\right)$ coupled only by the Coulomb interaction. In

the assumed long-wavelength limit $q_{x} \ell_{0} \ll 1$ we can approximate $f_{0, k_{x \alpha}-q_{x}, \pm}-f_{0, k_{x \alpha}, \pm}$ by $q_{x} \delta\left(k_{x \alpha}-k_{r 0}^{ \pm}\right)$and neglect the small shift $\propto q_{x} \ell_{0}$ in the argument of $\Psi_{0}\left(x-y_{0}\left(k_{x \alpha}\right)+\ell_{0}^{2} q_{x}\right)$, i.e., we can approximate the latter by $\Psi_{0}\left(x-y_{0}\left(k_{x \alpha}\right)\right)$. Then from Eqs. (16) and (17), after integration over $k_{x \alpha}$, we obtain

$$
\rho^{(l)}\left(\omega, q_{x}, y\right)=\frac{e^{2}}{\pi \hbar \epsilon} \frac{q_{x} \Psi_{0}^{2}\left(y-y_{r 0}^{+}\right)}{\omega-q_{x} v_{g 0}^{+}+i / \tau} \int_{-\infty}^{\infty} d \tilde{y} \int_{-\infty}^{\infty} d y^{\prime} \Psi_{0}^{2}\left(\tilde{y}-y_{r 0}^{+}\right) K_{l r}\left(\tilde{y}, y^{\prime} ; \omega, q_{x}\right)
$$

and

$$
\rho^{(r)}\left(\omega, q_{x}, y\right)=\frac{e^{2}}{\pi \hbar \epsilon} \frac{q_{x} \Psi_{0}^{2}\left(y-y_{r 0}^{-}\right)}{\omega-q_{x} v_{g 0}^{-}+i / \tau} \int_{-\infty}^{\infty} d \tilde{y} \int_{-\infty}^{\infty} d y^{\prime} \Psi_{0}^{2}\left(\tilde{y}-y_{r 0}^{-}\right) K_{r l}\left(\tilde{y}, y^{\prime} ; \omega, q_{x}\right)
$$

respectively. The solution of Eqs. (18) and (19) can be written as

$$
\rho^{(l)}\left(\omega, q_{x}, y\right)=\rho^{(l)}\left(\omega, q_{x}\right) \Psi_{0}^{2}\left(y-y_{r 0}^{+}\right), \quad \rho^{(r)}\left(\omega, q_{x}, y\right)=\rho^{(r)}\left(\omega, q_{x}\right) \Psi_{0}^{2}\left(y-y_{r 0}^{-}\right) .
$$

Since $X_{+}(z) \approx X_{l}(z)$ and $X_{-}(z) \approx X_{r}(z)$, we can consider $v_{g 0}^{+}$and $y_{r 0}^{+}$as parameters of the $l$ QW that are independent of those of the $r$ QW $v_{g 0}^{-}\left(y_{r 0}^{-}\right)$; the same holds for $E_{0,+}\left(k_{x}\right)$ and 
$E_{0,-}\left(k_{x}\right)$. Thus, in Eqs. (18)-(20) we can take, e.g., $\Delta y=y_{r 0}^{-}-y_{r 0}^{+} \gg \ell_{0}$. In particular, here we can assume different confining potentials in the $l$ and $r$ QWs, $V_{y}^{(l, r)}$.

Substituting Eq. (20) into Eqs. (18),(19) gives a system of two linear homogeneous equations for the unknowns $\rho^{(l, r)}\left(\omega, q_{x}\right)$. The condition of a nontrivial solution gives the dispersion relation (DR) for the renormalized, by the Coulomb coupling, single-well fundamental $\operatorname{EMP}\left(\omega_{\alpha_{z}}^{(c)}\left(q_{x}\right) \equiv \omega_{\alpha_{z}}^{(c)}\right)$ as

$$
\omega_{\alpha_{z}}^{(c)}=q_{x} V_{g+}-\frac{i}{\tau}+q_{x} a_{00}\left(q_{x}\right) \frac{e^{2}}{\pi \hbar \epsilon}-\alpha_{z} q_{x}\left[\left(\frac{e^{2}}{\pi \hbar \epsilon}\right)^{2} b_{00}^{2}\left(q_{x}, \Delta y, d\right)+V_{g-}^{2}\right]^{1 / 2},
$$

where $V_{g \pm}=\left(v_{g 0}^{-} \pm v_{g 0}^{+}\right) / 2$ and $\alpha_{z}= \pm$ corresponds to the \pm LSL. Here

$$
b_{00}\left(q_{x}, \Delta y, d\right)=\int_{-\infty}^{\infty} d x \int_{-\infty}^{\infty} d x^{\prime} \Psi_{0}^{2}(x) \Psi_{0}^{2}\left(x^{\prime}\right) K_{0}\left(\left|q_{x}\right| \sqrt{\left[\left(x-x^{\prime}\right)+\Delta y\right]^{2}+d^{2}}\right)
$$

and $a_{00}\left(q_{x}\right)=b_{00}\left(q_{x}, 0,0\right) \approx\left[\ln \left(1 /\left|q_{x}\right| \ell_{0}\right)+3 / 4\right]$ [17]; it is assumed that $v_{g 0}^{-} \geq v_{g 0}^{+}>0$. Without inter-QW Coulomb coupling, i.e., with $b_{00}\left(q_{x}, \Delta y, d\right)=0$ in Eq. (21), we obtain the DR of the fundamental EMPs for totally decoupled QWs as $\omega_{-}^{(r)}=q_{x} v_{g 0}^{-}+\left[e^{2} / \pi \hbar \epsilon\right] q_{x} a_{00}\left(q_{x}\right)-$ $i / \tau$, for the $r \mathrm{QW}$, and as $\omega_{+}^{(l)}=q_{x} v_{g 0}^{+}+\left[e^{2} / \pi \hbar \epsilon\right] q_{x} a_{00}\left(q_{x}\right)-i / \tau$, for the $l$ QW. If we neglect dissipation, this result coincides with that of Ref. [17] for $\nu=1$ in a single QW. For a GaAs-based DQW $(\epsilon=12.5)$ we have $2 e^{2} / \pi \hbar \epsilon \approx 10^{7} \mathrm{~cm} / \mathrm{sec}$ which is essentially larger than the typical $v_{g 0} \lesssim 10^{6} \mathrm{~cm} / \mathrm{sec}$, cf. [27], [17]. Moreover, for $D=\left(\Delta y^{2}+d^{2}\right)^{1 / 2} \leq 0.4 / q_{x} \gg \ell_{0}$ it follows that $b_{00}\left(q_{x}, \Delta y, d\right)>1$. Then for $q_{x} D \ll 1$ and $D^{2} / \ell_{0}^{2} \gg 1$ Eq. (21) gives the DR of the renormalized fundamental EMP of the $r$ QW, i.e., the fast EMP, as

$$
\omega_{-}^{(c)}=q_{x} V_{g+}+q_{x} \frac{e^{2}}{\pi \hbar \epsilon}\left[2 \ln \left(\frac{1}{\left|q_{x}\right| \ell_{0}}\right)-\ln \left(\frac{D}{\ell_{0}}\right)+0.85\right]-\frac{i}{\tau} ;
$$

and that of the $l$ QW, i.e., the slow EMP, as

$$
\omega_{+}^{(c)}=q_{x} V_{g+}+q_{x} \frac{e^{2}}{\pi \hbar \epsilon}\left[\ln \left(\frac{D}{\ell_{0}}\right)+0.65\right]-\frac{i}{\tau} .
$$

Substituting $\omega_{-}^{(c)}$ and $\omega_{+}^{(c)}$, given by Eqs. (23) and (24), into Eq. (18) we obtain $\rho^{(l)}\left(\omega_{-}^{(c)}, q_{x}\right) / \rho^{(r)}\left(\omega_{-}^{(c)}, q_{x}\right) \approx 1$ and $\rho^{(l)}\left(\omega_{+}^{(c)}, q_{x}\right) / \rho^{(r)}\left(\omega_{+}^{(c)}, q_{x}\right) \approx-1$, respectively. For $v_{g 0}^{-}=v_{g 0}^{+}$ these ratios are exactly 1 and -1 , respectively. Then, as can be seen from Eq. (20), the 
renormalized fundamental EMP of the $r$ QW, Eq. (23), shows an acoustic spatial behavior in the $y$ direction and that of the $l$ QW, Eq. (24), an optical one though its DR is purely acoustic.

For negligible dissipation the DRs (23) and (24) are shown in Fig. 1 by the solid $\left(D^{2} / \ell_{0}^{2}=\right.$ 3 ) and dashed $\left(D^{2} / \ell_{0}^{2}=10\right)$ curves, respectively, for $v_{g 0}^{-} \approx v_{g 0}^{+}=10^{6} \mathrm{~cm} / \mathrm{sec}, \epsilon=12.5$, and $\omega_{*}=e^{2} / \pi \hbar \epsilon \ell_{0}$. The dotted curve shows the DR of an isolated, single-well fundamental EMP $\omega_{-}^{(l)}=\omega_{+}^{(r)}=q_{x} v_{g 0}^{-}+\left[e^{2} / \pi \hbar \epsilon\right] q_{x}\left[\ln \left(1 /\left(\left|q_{x}\right| \ell_{0}\right)\right)+3 / 4\right]$. The upper solid and dashed curves represent the fast EMP and the lower ones the slow EMP.

\section{B. Strong tunnel coupling}

We now consider case (ii) in which $2|T| \gtrsim \Delta$ holds. As in Sec. III A, we make the approximations $f_{0, k_{x \alpha}-q_{x}, \pm}-f_{0, k_{x \alpha}, \pm} \approx q_{x} \delta\left(k_{x \alpha}-k_{r 0}^{ \pm}\right)$and $\Psi_{0}\left(x-y_{0}\left(k_{x \alpha}\right)+\ell_{0}^{2} q_{x}\right) \approx \Psi_{0}(x-$ $\left.y_{0}\left(k_{x \alpha}\right)\right)$. Then integration over $k_{x \alpha}$ in Eq. (15) results in

$$
\begin{aligned}
\rho^{(j)}\left(\omega, q_{x}, y\right) & =q_{x} \frac{e^{2}}{\pi \hbar \epsilon} \sum_{\alpha_{z}= \pm} \frac{<\alpha_{z} \mid \alpha_{z}>_{j}}{\omega-q_{x} v_{g 0}^{\alpha_{z}}+i / \tau} \Psi_{0}^{2}\left(y-y_{r 0}^{\alpha_{z}}\right) \\
& \times \int_{-\infty}^{\infty} d \tilde{y} \int_{-\infty}^{\infty} d y^{\prime} \Psi_{0}^{2}\left(\tilde{y}-y_{r 0}^{\alpha_{z}}\right)\left\{\left[<\alpha_{z} \mid \alpha_{z}>_{l} K_{l r}\left(\tilde{y}, y^{\prime} ; \omega, q_{x}\right)\right]+[r \leftrightarrow l]\right\},
\end{aligned}
$$

where $j=l, r$. The solution of Eqs. (25) can be written as

$$
\rho^{(i)}\left(\omega, q_{x}, y\right)=\sum_{\alpha_{z}= \pm}<\alpha_{z} \mid \alpha_{z}>_{i} \rho_{\alpha_{z}}\left(\omega, q_{x}\right) \Psi_{0}^{2}\left(y-y_{r 0}^{\alpha_{z}}\right)
$$

where $i=l, r$. Substituting Eq. (26) into Eq. (25) and demanding that the factors in front of $\Psi_{0}^{2}\left(y-y_{r 0}^{ \pm}\right)$on both sides of Eq. (25) be equal for $j=l$ and for $j=r$, leads to a system of two linear equations for $\rho_{ \pm}\left(\omega, q_{x}\right)$ written as

$$
\begin{aligned}
& \rho_{\alpha_{z}}\left(\omega, q_{x}\right)\left\{\frac{\pi \hbar \epsilon}{e^{2} q_{x}}\left(\omega-q_{x} v_{g 0}^{\alpha_{z}}+i / \tau\right)-N^{4}\left[\left(1+M^{-8}\right) a_{00}\left(q_{x}\right)+2 M^{-4} b_{00}\left(q_{x}, 0, d\right)\right]\right\} \\
& -\rho_{-\alpha_{z}}\left(\omega, q_{x}\right) N^{4}\left\{\left[1+M^{-8}\right] b_{00}\left(q_{x}, \Delta y, d\right)+2 M^{-4} b_{00}\left(q_{x}, \Delta y, 0\right)\right\}=0 .
\end{aligned}
$$


Here $N^{2}=\left(\Delta+\Delta_{T}\right) / 2 \Delta_{T}, M^{2}=\left(\Delta+\Delta_{T}\right) / 2 T, \alpha_{z}=-$ for the first equation and $\alpha_{z}=+$ for the second one. The condition for a nontrivial solution of these equations gives the DR of the renormalized, due to tunneling and Coulomb coupling, single-well fundamental EMP

$$
\begin{aligned}
\omega_{\alpha_{z}}^{(t c)}= & q_{x} V_{g+}-\frac{i}{\tau}+q_{x} \frac{e^{2}}{\pi \hbar \epsilon} N^{4}\left\{\left[1+M^{-8}\right] a_{00}\left(q_{x}\right)+2 M^{-4} b_{00}\left(q_{x}, 0, d\right)\right\} \\
& -\alpha_{z} q_{x}\left\{\left(\frac{e^{2}}{\pi \hbar \epsilon}\right)^{2} N^{8}\left[\left(1+M^{-8}\right) b_{00}\left(q_{x}, \Delta y, d\right)+2 M^{-4} b_{00}\left(q_{x}, \Delta y, 0\right)\right]^{2}+V_{g-}^{2}\right\}^{1 / 2} .
\end{aligned}
$$

Notice that for finite $\Delta$ and $T \rightarrow 0$, i.e., without tunnel coupling, Eq. (28) coincides with Eq. (21). Further, the first term under the square root in Eq. (28) is typically much larger than the second one. Then

$$
\begin{gathered}
\omega_{\alpha_{z}}^{(t c)=} q_{x} V_{g+}-\frac{i}{\tau}+q_{x} \frac{e^{2}}{\pi \hbar \epsilon}\left[a_{00}\left(q_{x}\right)-\alpha_{z} b_{00}\left(q_{x}, \Delta y, d\right)\right] \\
-q_{x} \frac{2 e^{2}}{\pi \hbar \epsilon} \frac{T^{2}}{\Delta_{T}^{2}}\left[a_{00}\left(q_{x}\right)-b_{00}\left(q_{x}, 0, d\right)\right] ;
\end{gathered}
$$

on the RHS we have neglected the cotribution $\alpha_{z}\left[b_{00}\left(q_{x}, \Delta y, 0\right)-b_{00}\left(q_{x}, \Delta y, d\right)\right]$, which is small in comparison with $\left[a_{00}\left(q_{x}\right)-b_{00}\left(q_{x}, 0, d\right)\right]$ due to the assumed condition $\Delta y^{2} / d^{2} \gg 1$. Now in the actual region $1 \geq d / \ell_{0} \geq 1 / 4$ we numerically evaluate $\left[a_{00}\left(q_{x}\right)-b_{00}\left(q_{x}, 0, d\right)\right] \approx d / \ell_{0}$ with relative precision $\leq 10 \%$. Then Eq. (29) gives the DR of the fast EMP as

$$
\omega_{-}^{(t c)}=q_{x} V_{g+}+q_{x} \frac{e^{2}}{\pi \hbar \epsilon}\left[2 \ln \left(\frac{1}{\left|q_{x}\right| \ell_{0}}\right)-\ln \left(\frac{D}{\ell_{0}}\right)+0.85-\frac{2 d}{\ell_{0}} \frac{T^{2}}{\Delta^{2}+4 T^{2}}\right]-\frac{i}{\tau},
$$

and that of the slow EMP as

$$
\omega_{+}^{(t c)}=q_{x} V_{g+}+q_{x} \frac{e^{2}}{\pi \hbar \epsilon}\left[\ln \left(\frac{D}{\ell_{0}}\right)+0.65-\frac{2 d}{\ell_{0}} \frac{T^{2}}{\Delta^{2}+4 T^{2}}\right]-\frac{i}{\tau},
$$

where $\Delta y / \ell_{0}=\ell_{0}\left[k_{e}^{0,-}-k_{e}^{0,+}\right] \approx\left(\omega_{c} / \Omega\right)\left[\left(\Delta^{2}+4 T^{2}\right) / 2 \hbar \omega_{c} \Delta_{F 0}\right]^{1 / 2}$ for $\Delta_{F 0}^{ \pm}=\Delta_{F 0} \mp \Delta_{T} / 2$ and $\Delta_{F 0} \gg \Delta_{T} / 2$. Using Eqs. (30), (31), and (27) we obtain $\rho_{-}\left(\omega_{-}^{(t c)}, q_{x}\right) / \rho_{+}\left(\omega_{-}^{(t c)}, q_{x}\right)=1$ for the fast mode and $\rho_{-}\left(\omega_{+}^{(t c)}, q_{x}\right) / \rho_{+}\left(\omega_{+}^{(t c)}, q_{x}\right)=-1$ for the slow mode. The corresponding charge density profiles are obtained from Eq. (26) as 


$$
\begin{aligned}
& \frac{\rho^{(l)}\left(\omega_{\mp}^{(t c)}, q_{x}, y\right)}{\rho_{-}\left(\omega_{\mp}^{(t c)}, q_{x}\right)}=N^{2}\left\{M^{-4} \Psi_{0}^{2}\left(y-y_{r 0}^{-}\right) \pm \Psi_{0}^{2}\left(y-y_{r 0}^{+}\right)\right\} \\
& \frac{\rho^{(r)}\left(\omega_{\mp}^{(t c)}, q_{x}, y\right)}{\rho_{-}\left(\omega_{\mp}^{(t c)}, q_{x}\right)}=N^{2}\left\{\Psi_{0}^{2}\left(y-y_{r 0}^{-}\right) \pm M^{-4} \Psi_{0}^{2}\left(y-y_{r 0}^{+}\right)\right\},
\end{aligned}
$$

where the upper (lower) sign corresponds to the fast (slow) mode.

For strong tunnel coupling $\Delta \lesssim 2|T|=1 \mathrm{meV}$ we plot in Fig. 2 the group velocities of both DQW fundamental EMPs, obtained from Eqs. (30) and (31), as function of $\Delta$ with $v_{*}=e^{2} / \pi \hbar \epsilon$ and $\Delta_{*}=\sqrt{3} \mathrm{meV} . \Delta$ can be changed experimentally by a transverse electric field applied to the DQW structure. Notice that the group velocity of the fast mode, $\partial \omega_{-}^{(t c)} / \partial q_{x}$, depends on $q_{x}$ whereas that of the slow mode, $\partial \omega_{+}^{(t c)}\left(q_{x}\right) / \partial q_{x}$, does not. For a GaAs-based DQW, $\Omega=7.8 \times 10^{11} \sec ^{-1}$ [27], and $B=9$ Tesla we have $\omega_{c} / \Omega \approx 30$, and $\Delta_{F 0}=4 \mathrm{meV}$. The value of $d / \ell_{0}$ is 0.5 for the solid and dotted curves and 1 for the dashed curves. The upper solid and dashed curves represent the group velocity of the fast EMP for $q_{x} \ell_{0}=10^{-3}$ and the lower ones that of the slow EMP. The dotted curve represents the group velocity of the fast DQW fundamental EMP for $q_{x} \ell_{0}=10^{-2}$. For $\Delta=0$ we have $\Delta y / \ell_{0} \approx 2.7$ $\left(\Delta_{T} / 2 \Delta_{F 0}=1 / 8 \ll 1\right)$ and $\Delta y / \ell_{0} \approx 5.4$ for $\Delta=\sqrt{3} \mathrm{meV}\left(\Delta_{T} / 2 \Delta_{F 0}=1 / 4 \ll 1\right)$. Thus the assumed conditions $(\Delta y / d)^{2} \gg 1$, etc., are well satisfied in Fig. 2. In addition, we have $v_{g 0}^{ \pm} \approx 5 \times 10^{5}\left[1 \mp \Delta_{T} /\left(4 \Delta_{F 0}\right)\right] \mathrm{cm} / \mathrm{sec}$ and the term $q_{x}\left(v_{g 0}^{-}+v_{g 0}^{+}\right) / 2$ is independent of $\Delta$ or T. As is clear from Fig. 2, the change of $\Delta / \Delta_{*}$ from 0 to 1 changes the group velocities of both DQW fundamental EMPs appreciably and especially that of the slow one which shows a 1.78 times increase of its group velocity within that region. Finally, for larger $d / \ell_{0}$ the group velocity of the slow mode shows a stronger dependence on $\Delta$ (for small $\Delta$ ) whereas that of the fast mode shows a weaker one.

\section{DISCUSSION AND CONCLUDING REMARKS}

The above treatment has shown that in a typical DQW the Coulomb and tunnel coupling lead to two new DQW fundamental EMPs whose dispersion laws and spatial structure, along 
the $y$-axis, are essentially different than those of single-well fundamental EMPs. These singlewell EMPs follow from the former if we neglect inter-well coupling. The fast EMP behaves as $\omega \propto q_{x} \ln \left(1 / q_{x}\right)$ whereas the slow EMP behaves as $\omega \propto q_{x}$. The latter has an acoustic dispersion law though its spatial structure, along the $y$ axis, is optical. Indeed, for both negligible and strong tunneling, when the DQW levels are in resonance and $\Delta$ vanishes, the charge localized at the edge $y_{r 0}^{-}$of the - LSL has exactly the opposite value of that localized at the edge $y_{r 0}^{+}$of the + LSL where $\Delta y=y_{r 0}^{-}-y_{r 0}^{+} \gg \ell_{0}$. The group velocity of the slow EMP is much smaller than that of the fast EMP. Moreover, they can be easily modulated by applying a transverse electric field to the DQW structure in the case of strong tunnel coupling. This renormalization of single-well fundamental EMPs is essentially different than that of the right- and left-edge fundamental EMPs in a single-well 2DEG channel with relatively short width, $q_{x} W \ll 1$. As shown in Ref. [1], in the latter case the renormalized EMPs have dispersion laws $\omega \propto \pm q_{x} \sqrt{\ln \left(1 / q_{x}\right)}$ that give the same absolute value for a group velocity.

We have neglected the possible spatial inhomogeneity of the background dielectric constant $\epsilon$ along the $z$ direction. In GaAlAs/GaAs QWs such an inhomogeneity is relatively small. Within the RPA we can include the effect of scattering only in a phenomenological manner through the small parameter $1 / \tau$. This effect can be treated approximately along the lines of Refs. [17] and [21]. We have also neglected the possible screening influence of the gates or other free charges outside the spacer layer. Their influence on the slow mode, if they are at a distance much greater than $\Delta y$ far from $y_{r 0}^{ \pm}$, should be negligible. We have also assumed that the confining potential, flat in the interior of the channel, is smooth on the magnetic length scale but sufficiently steep at the edges that Landau-level flattening [28] can be neglected [29]. In addition, though the thicknesses $d_{l}$ and $d_{r}$ of the QWs have been neglected, we believe that this often used approximation 22] does not affect our results essentially.

Note added in proof. Remarkably the results of Sec. III A partly overlap with the $M=1$ results of Ref. 30, obtained in the time-dependent Hartree-Fock approximation for 
the particular $M=1$ edge excitations of a double quantum-dot system with zero tunneling between the dots and total $\nu=2$ in the limit of large dots. In this limit the dot radius $R$ is large compared to both the magnetic length $\ell_{0}$ and the dot separation $d$. Then the energy

of the in-phase collective magnetoplasmon excitations $E_{m p l}^{+}$is given by Eq. (29) of Ref. 30 and that of the out-of-phase excitations $E_{m p l}^{-}$by Eq. (30) of Ref. 30. The former follows from Eq. (23) and the latter from Eq. (24) of Sec. III A if we make the changes $q_{x} \rightarrow 1 / R$, $\epsilon \rightarrow 1$, and neglect $q_{x} V_{g+}$ and the important terms 0.85 in Eq. (23) and 0.65 in Eq. (24) .

\section{ACKNOWLEDGMENTS}

We thank Dr. A. H. MacDonald for drawing our attention to Ref. [30]. This work was supported by the Canadian NSERC Grant No. OGP0121756. O G B acknowledges partial support by the Ukrainian SFFI Grant No. 2.4/665. 


\section{REFERENCES}

[1] V. A. Volkov and S. A. Mikhailov, Zh. Eksp. Teor. Fiz. 94, 217 (1988) [Sov. Phys. JETP 67, 1639 (1988)].

[2] M. Wassermeier, J. Oshinowo, J. P. Kotthaus, A. H. MacDonald, C. T. Foxon, and J. J. Harris, Phys. Rev. B 41, 10287 (1990).

[3] I. Grodnensky, D. Heitmann, and K. von Klitzing, Phys. Rev. Lett. 67,1019 (1991).

[4] X. G. Wen, Phys. Rev. B 43, 11025 (1991).

[5] M. Stone, Ann. Phys. (NY) 207, 38 (1991).

[6] M. Stone, H. W. Wyld, and R. L. Schult, Phys. Rev. B 45, 14156 (1992).

[7] R. C. Ashoori, H. L. Stormer, L. N. Pfeiffer, K. W. Baldwin, and K. West, Phys. Rev. B 45, 3894 (1992).

[8] V. I. Tal'yanskii, A. V. Polisski, D. D. Arnone, M. Pepper, C. G. Smith, D. A. Ritchie, J. E. Frost, and G. A. C. Jones, Phys. Rev. B 46, 12427 (1992).

[9] N. B. Zhitenev, R. J. Haug, K. von Klitzing, and K. Eberl, Phys. Rev. Lett. 71, 2292 (1993); Phys. Rev. B 49, 7809 (1994).

[10] I. L. Aleiner and L. I. Glazman, Phys. Rev. Lett. 72, 2935 (1994).

[11] C. de Chamon and X. G. Wen, Phys. Rev. B 49, 8227 (1994).

[12] J. S. Giovanazzi, L. Pitaevskii, and S. Stringari, Phys. Rev. Lett. 72, 3230 (1994).

[13] G. Ernst, R. J. Haug, J. Kuhl, K. von Klitzing, and K. Eberl, Phys. Rev. Lett. 77, 4245 (1996).

[14] J. H. Han and D. J. Thouless, Phys. Rev. B 55, R1926 (1997).

[15] U. Zulicke, R. Bluhm, V. A. Kostelcky, and A. H. MacDonald, Phys. Rev. B 55, 9800 (1997). 
[16] O. G. Balev and P. Vasilopoulos, Phys. Rev. Lett., 81, 1481 (1998).

[17] O. G. Balev and P. Vasilopoulos, Phys. Rev. B 56, 13252 (1997).

[18] Kelvin, William Thomson, Phil. Mag. x 97 (1880).

[19] O. G. Balev and P. Vasilopoulos, Phys. Rev. B, in press.

[20] F. T. Vasko, O. G. Balev, and P. Vasilopoulos, Phys. Rev. B 47, 16433 (1993).

[21] O. G. Balev and P. Vasilopoulos, Phys. Rev. B 54, 4863 (1996).

[22] A. H. MacDonald, P. M. Platzman, and G. S. Boebinger, Phys. Rev. Lett. 65, 775 (1990).

[23] G. S. Boebinger, H. W. Jiang, L. N. Pfeiffer, and K. W. West, Phys. Rev. Lett. 64, $1793(1990)$.

[24] P. M. Platzmann and P. A. Wolff, Waves and Interactions in Solid State Plasmas, Solid State Physics, Eds. H. Ehrenreich, F. Seitz and D. Turnbull, Suppl. 13, Academic Press, Ney York and London (1973).

[25] O. G. Balev, Fiz. Tverd. Tela (Leningrad). 32, 871 (1990) [Sov. Phys. Solid State 32, $514(1990)]$.

[26] O. G. Balev and P. Vasilopoulos, Phys. Rev. B 56, 6748 (1997).

[27] G. Muller, D. Weiss, A. V. Khaetskii, K. von Klitzing, S. Koch, H. Nickel, W. Schlapp, and R. Losch, Phys. Rev. B 45, 3932 (1992).

[28] D. B. Chklovskii, B. I. Shklovskii, and L. I. Glazman, Phys. Rev. B 46, 4026 (1992).

[29] L. Brey, J. J. Palacios, and C. Tejedor, Phys. Rev. B 47, 13884 (1993); T. Suzuki, Tsuneya Ando, J. Phys. Soc. Jpn. 62, 2986 (1993).

[30] J. Hu, E. Dagotto, and A. H. MacDonald, Phys. Rev. B 54, 8616 (1996). 


\section{FIGURES}

FIG. 1. Dispersion relation of the DQW fundamental EMPs, for negligible tunnel coupling $2|T| / \Delta \ll 1$. The solid curves are for $D^{2} / \ell_{0}^{2}=3$ and dashed ones for $D^{2} / \ell_{0}^{2}=10 ; \omega_{*}=e^{2} /\left(\pi \hbar \epsilon \ell_{0}\right)$. The dotted curve is the single-well fundamental EMP without inter-well Coulomb coupling. The upper solid and dashed curves represent the fast EMP and the lower ones the slow EMP.

FIG. 2. Group velocity of the DQW fundamental EMPs for strong tunnel coupling, $\Delta \leq 2|T|=1 \mathrm{meV}$, as a function of $\Delta ; v_{*}=e^{2} /(\pi \hbar \epsilon)$ and $\Delta_{*}=\sqrt{3} \mathrm{meV}$. The value of $d / \ell_{0}$ is 0.5 for the solid and dotted curves and 1 for the dashed curves. The upper solid and dashed curves represent the fast EMP, for $q_{x} \ell_{0}=10^{-3}$, and the lower ones the slow EMP, whose velocity is independent of $q_{x}$. The dotted curve represents the fast EMP for $q_{x} \ell_{0}=10^{-2}$. 


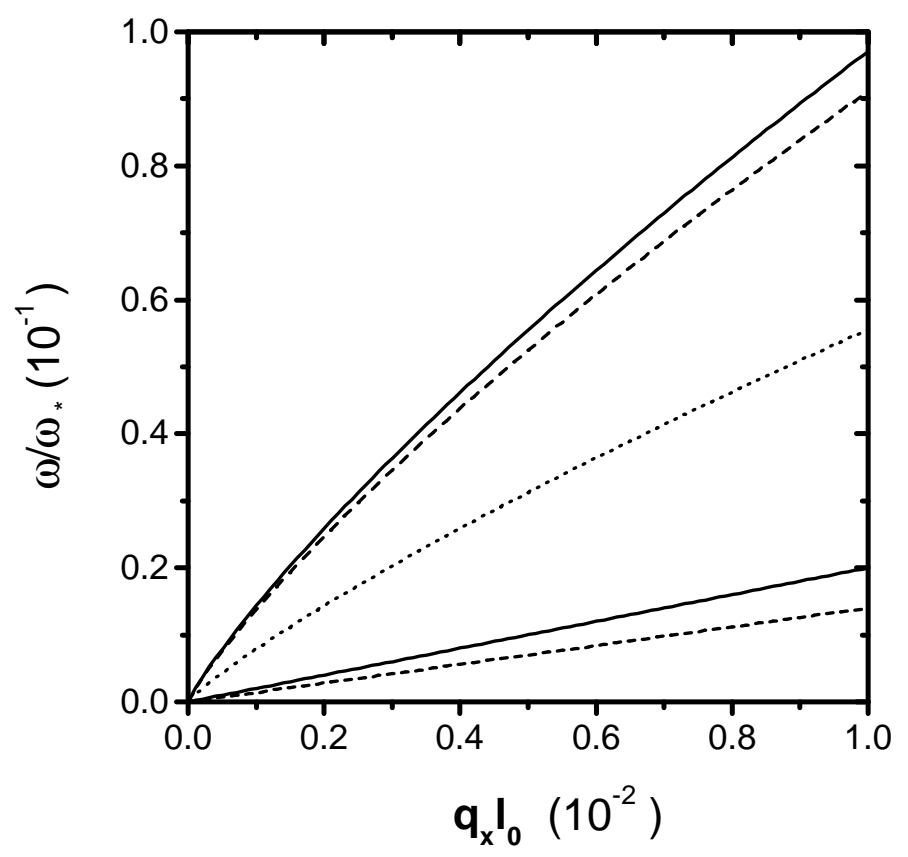

Fig. 1, Balev et. al. 


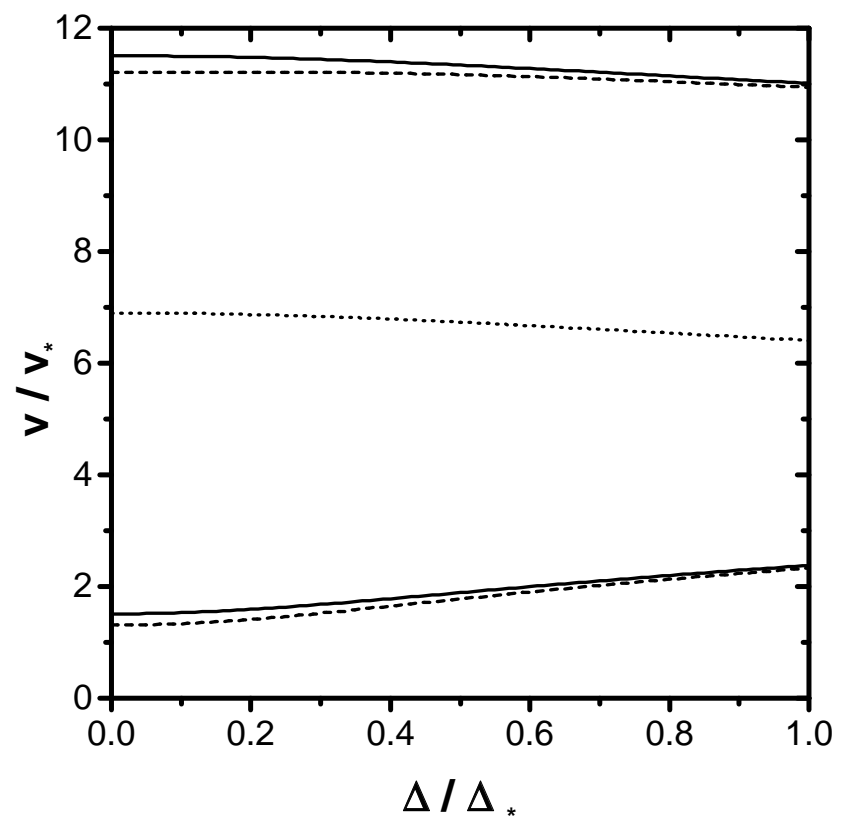

Fig. 2, Balev et al. 